\title{
BeppoSAX observation of 4U 1705-44: detection of hard X-ray emission in the soft state
}

\author{
S. Piraino ${ }^{1,2}$, A. Santangelo ${ }^{2}$, T. Di Salvo ${ }^{3}$, P. Kaaret ${ }^{4}$, D. Horns ${ }^{2}$, R. Iaria ${ }^{3}$, and L. Burderi ${ }^{5}$ \\ 1 INAF - IASF di Palermo, via Ugo La Malfa 153, 90146 Palermo, Italy \\ e-mail: Santina.Piraino@ifc.inaf.it \\ 2 IAAT, University of Tübingen, Sand 1, 72076 Tübingen, Germany \\ 3 Dipartimento di Scienze Fisiche ed Astronomiche, Università di Palermo, via Archirafi 36, 90123 Palermo, Italy \\ ${ }^{4}$ Department of Physics and Astronomy, University of Iowa, Iowa City, IA 52246, USA \\ 5 Universitá degli Studi di Cagliari, Dipartimento di Fisica, SP Monserrato-Sestu, KM 0.7, 09042 Monserrato, Italy
}

Received 10 May 2007 / Accepted 19 June 2007

\begin{abstract}
Context. 4U 1705-44 is one of the best-studied type I X-ray burster and atoll sources.

Aims. Since it covers a wide range in luminosity (from a few to $50 \times 10^{36} \mathrm{erg} \mathrm{s}^{-1}$ ) and shows clear spectral state transitions, it represents a good laboratory for testing the accretion models proposed for atoll sources.

Methods. We analyzed the energy spectrum accumulated with BeppoSAX observations (43.5 ks) in August 2000 when the source was in a soft spectral state.

Results. The continuum of the wide-band energy spectrum is well-described by the sum of a blackbody $\left(k T_{\mathrm{bb}} \sim 0.56 \mathrm{keV}\right)$ and a Comptonized component (seed-photon temperature $k T_{\mathrm{W}} \sim 1 \mathrm{keV}$, electron temperature $k T_{\mathrm{e}} \sim 2.7 \mathrm{keV}$, and optical depth $\tau \sim 11$ ). A hard tail was detected at energies above $\sim 25 \mathrm{keV}$. The latter can be modeled by a power law having a photon index $\sim 2.9$, which contributes $\sim 11 \%$ of the total flux in the range $0.1-200 \mathrm{keV}$. A broad emission line, possibly from a relativistic accretion disk, models the feature in the $\mathrm{Fe} \mathrm{K}$ line region of the spectrum.

Conclusions. This is the first time that a high-energy tail has been observed during a soft state of the source.
\end{abstract}

Key words. accretion, accretion disks - stars: individual: 4U 1705-44 - stars: neutron - X-rays: stars - X-rays: binaries X-rays: general

\section{Introduction}

Numerous similarities have been found between low-mass X-ray binaries containing neutron stars and accreting stellar-mass black holes. The similarity between the X-ray properties of these systems suggests that the fundamental physical processes producing X-ray emission must be quite similar. Indeed, neutron stars and black holes have very similar gravitational potentials, and the neutron star radius is close to the size of the last stable orbit of material around a black hole, so the properties of the accretion flow around the compact object should be similar. On the other hand, since neutron stars have a solid surface, unlike black holes, they should show unique signatures. X-ray bursts and coherent pulsations are unique signatures of neutron stars. However, they are a sufficient, but not necessary condition, for the presence of a solid surface, since not all neutron star systems show these features.

There have been attempt to look for effects of the stellar surface on the energy spectrum by comparing black holes and neutron stars in similar luminosity states. The proposed black hole spectral features, such as an ultra-soft spectral state at high luminosity, hard X-ray emission at low luminosity, and steep X-ray tail seen at high luminosity, have been contradicted by counter-examples found in neutron-star spectra (see e.g. Tanaka \& Lewin 1995; Barret \& Vedrenne 1994; Di Salvo et al. 2001). A unique black hole spectral signature has remained so far elusive.
Hasinger \& van der Klis (1989) divided the low-mass X-ray binaries containing neutron stars into two groups called $\mathrm{Z}$ sources and atoll sources after the patterns these sources trace out in the X-ray color-color diagram (CD). The $\mathrm{CD}$ of $\mathrm{Z}$ sources displays a Z-like track, whereas the $\mathrm{CD}$ of atoll sources show a C-like track where two branches can be identified, the island and the banana states. The island state is characterized by lower count rates, slower motion in CD and stronger band-limited noise than the banana state. For both classes, the position of the source in the CD is thought to be an indicator of the mass accretion rate $(\dot{M})$ increasing from the upper left to the bottom right of the $\mathrm{Z}$ track for $\mathrm{Z}$ sources and from the island state to the right of the horizontally elongated banana state for atoll sources. The $\mathrm{Z}$ sources are thought to have larger magnetic fields and/or higher $\dot{M}$ than atoll sources.

Recently, it has been shown that after sampling the source intensity states of atoll sources enough long, the shape of their CD tends to resemble those of $\mathrm{Z}$ sources (Gierlínski \& Done 2002; Muno et al. 2002). However, the similarities of the atoll CD track with that of $\mathrm{Z}$ sources are mainly confined to the banana branch. Moreover, the properties of atoll sources along the CD track are very different from those shown by $\mathrm{Z}$ sources (e.g. Barret \& Olive 2002; van der Klis 2006).

The bright low-mass X-ray binary 4U 1705-44 was classified by Hasinger \& van der Klis (1989) as an atoll source. Long-term monitoring of this source succeeded in sampling 
the complete track in the CD (Gierlínski \& Done 2002; Muno et al. 2002; Barret \& Olive 2002).

$4 \mathrm{U} 1705-44$ is an X-ray burster (Sztajno et al. 1985), whose bursting activity and frequency depend on the persistent emission. During the low state, when type I X-ray bursts are most frequent, the spectrum is unusually hard (Langmeier et al. 1987).

In this Letter, we report on measurements with the broad band coverage, sensitivity, and good energy resolution of BeppoSAX (Boella et al. 1997a) of the 4U 1705-44 X-ray spectrum obtained in the energy range $0.3-200 \mathrm{keV}$ at the beginning of a clear transition from a high to a low flux level.

\section{Observations}

We performed a BeppoSAX observation of $4 \mathrm{U} 1705-44$, on August 20-21, 2000 (MJDs = 51766/7) for a total of $43.5 \mathrm{ksec}$ of on-source observing time. Here, we report on the results of the average energy spectrum. Details on the timing and spectral evolution along the color-color diagram will be reported in a subsequent paper (Piraino et al. 2007, in preparation).

We obtained spectra from the four BeppoSAX Narrow Field Instruments (NFIs) in energy bands selected to give a good signal-to-noise ratio for this source: the Low Energy Concentrator Spectrometer (LECS, 0.3-4 keV; Parmar et al. 1997), the Medium Energy Concentrator Spectrometer (MECS, 2-10 keV; Boella et al. 1997b), the High Pressure Proportional Gas Scintillation Counter (HPGSPC, 8-50 keV; Manzo et al. 1997), and the Phoswich Detection System (PDS, 15-200 keV; Frontera et al. 1997). The LECS and MECS data were extracted in circular regions centered on the source position using radii of $8^{\prime}$ and $4^{\prime}$, respectively, containing $95 \%$ of the source flux. Identical circular regions in blank field data were used for the extraction of background spectra and background subtraction. As the source lies in the direction of the Galactic bulge, we scaled the blank field background at the source position to the mean level of the background around the source during the observation using a factor of 2.7 for LECS and 2.64 for MECS. Spectra accumulated from Dark Earth data and during off-source intervals were used for the background subtraction for HPGSPC and PDS spectra, respectively.

The LECS and MECS spectra were rebinned to sample the instrument energy resolution with the same number of channels at all energies, and the HPGSPC and PDS spectra were grouped using a logarithmic grid. The spectral analysis was performed with the data analysis package XSPEC v. 11.2 (Arnaud 1996). A normalization factor has been included to account for the mismatch in the BeppoSAX instruments' absolute flux calibration. We found relative normalizations in good agreement with typically observed values (Fiore et al. 1999).

\section{Spectral analysis}

We fit the 0.3-200 keV BeppoSAX spectrum of 4U 170544 with several single or multicomponent continuum models. The combination of the comptt Comptonization model (Titarchuk 1994) plus blackbody gave a fit having $\chi^{2} /$ d.o.f. $=$ $964.3 / 530\left(\chi_{v}^{2} \simeq 1.82\right)$. Using a multicolor disk blackbody, diskbb, instead of a simple blackbody, we obtained a $\chi^{2} /$ d.o.f. $=1077 / 530\left(\chi_{v}^{2} \simeq 2.03\right)$.

The fit was improved by adding a broad-iron K-line feature. Using a simple Gaussian line at $6.58 \pm 0.05 \mathrm{keV}$ $(\sigma=0.31 \pm 0.08 \mathrm{keV}, E W=63 \mathrm{eV})$, the fit gave a $\chi^{2} /$ d.o.f. $=$ $701.28 / 527\left(\chi_{v}^{2} \simeq 1.33\right)$. Line emission from a relativistic accretion disk (diskline, Fabian et al. 1989) at $\simeq 6.7 \mathrm{keV}$ works
Table 1. Results of fitting the $4 \mathrm{U} 1705-44$ spectra in the energy band $0.3-200 \mathrm{keV}$.

\begin{tabular}{l|c}
\hline \hline Parameter & Value \\
\hline$N_{\mathrm{H}}\left(\times 10^{22} \mathrm{~cm}^{-2}\right)$ & $1.9 \pm 0.1$ \\
$k T_{\mathrm{bb}}(\mathrm{keV})$ & $0.56 \pm 0.01$ \\
$N_{\mathrm{bb}}(\mathrm{keV})$ & $(2.23 \pm 0.11) \times 10^{-2}$ \\
$k T_{\mathrm{W}}(\mathrm{keV})$ & $1.13_{-0.02}^{+0.05}$ \\
$k T_{\mathrm{e}}(\mathrm{keV})$ & $2.72 \pm 0.09$ \\
$\tau$ & $11.0 \pm 0.6$ \\
$N_{\mathrm{c}}$ & $0.35 \pm 0.02$ \\
$E_{\mathrm{Fe}}(\mathrm{keV})$ & $6.7_{-0.5}^{+0.2}$ \\
$R_{\text {in }}\left(R_{\mathrm{g}}\right)$ & $8.1_{-2.1}^{+4.2}$ \\
Incl $(\mathrm{deg})$ & $28_{-8}^{+20}$ \\
$I_{\mathrm{Fe}}\left(\mathrm{ph} \mathrm{cm}{ }^{-2} \mathrm{~s}^{-1}\right)$ & $\left(4.7_{-0.6}^{+2.0}\right) \times 10^{-3}$ \\
Fe $E q . W .(\mathrm{eV})$ & 109 \\
$\Gamma$ & $2.9_{-0.3}^{+0.2}$ \\
$N_{p}$ & $0.7_{-0.2}^{+0.4}$ \\
$F_{\text {totab }}$ & 5.81 \\
$F_{\text {tot }}$ & 16.7 \\
$\chi_{\text {red }}^{2} /$ d.o.f. & $1.183 / 524$ \\
\hline
\end{tabular}

Note - The model consists of blackbody, Comptonization comptt, and power-law components, and a diskline emission line, all subject to interstellar absorption. Uncertainties are at the $90 \%$ confidence level for a single parameter. The total flux is in the $0.1-100 \mathrm{keV}$ energy range and in units of $\times 10^{-9} \mathrm{ergs}^{-2} \mathrm{~s}^{-1}$.

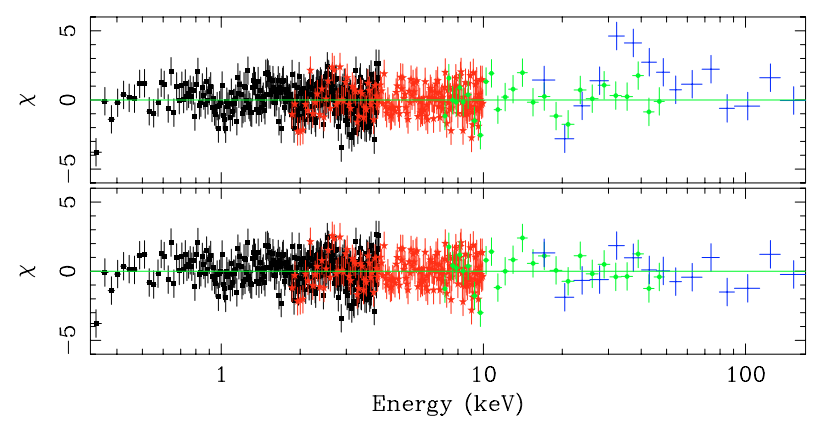

Fig. 1. Residuals in units of $\sigma$ with respect to the best-fit model without (upper panel) and with (bottom panel) the power-law component.

slightly better with $\chi^{2} /$ d.o.f. $=678.75 / 526\left(\chi_{v}^{2} \simeq 1.29\right)$. We report the best-fit parameters in Table 1 . An excess of the counts above $\sim 25 \mathrm{keV}$ was apparent in the spectrum. None of the twocomponent continuum models we tried could model this excess. By adding a power-law with a photon index 2.9 to the blackbody+comptt continuum model, the fit improved significantly $\left(\chi^{2} /\right.$ d.o.f. $=620 / 524, \chi_{v}^{2} \simeq 1.18$; an F-test gave a probability of chance improvement of the fit of $\simeq 10^{-10}$ ). This component contributed $\sim 11 \%$ of the $0.1-200 \mathrm{keV}$ source flux. The residuals after including a power-law component are shown in the bottom panel of Fig. 1. The average spectrum, together with the total model and its components, is shown in Fig. 2.

Note that Fiocchi et al. (2007) present a spectral analysis of INTEGRAL and BeppoSAX data of $4 \mathrm{U}$ 1705-44, which includes this BeppoSAX observation, claiming the detection of a Compton reflection component. However they did not include HPGSPC data in their analysis. Using their model in the whole BeppoSAX $(0.1-200 \mathrm{keV})$ range, we obtain a $\chi^{2} /$ d.o.f. $=$ $715.9 / 526$ and evident residuals in the $10-50 \mathrm{keV}$ range. We 


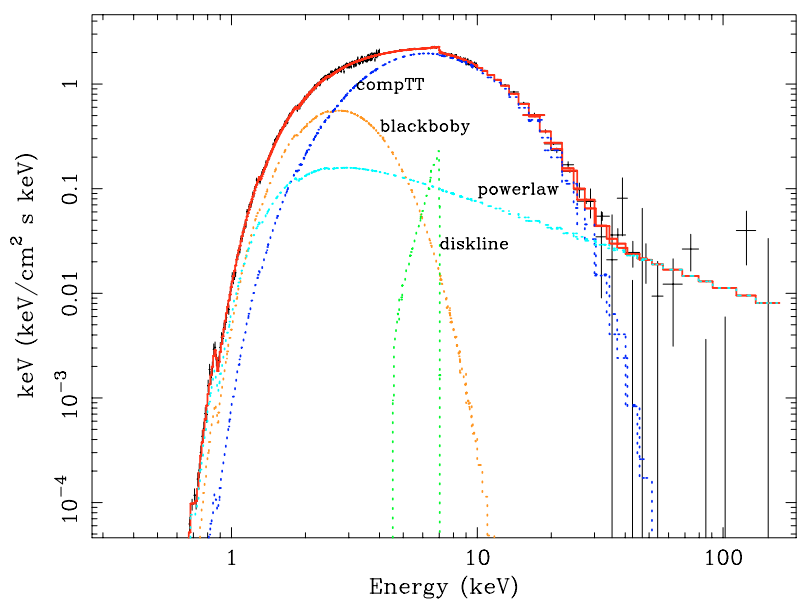

Fig. 2. X-ray (unfolded) spectrum together with the best-fit model (red line) and its components: blackbody (orange), Comptonization (blue), power-law (blue-green), diskline (green).

therefore conclude that the model presented here describes the energy spectrum of the source better in the entire BeppoSAX band.

\section{Discussion}

We have analyzed a BeppoSAX observation of the atoll source and X-ray burster $4 \mathrm{U} 1705-44$. The observation was performed in August 2000 for a total exposure time of $43.5 \mathrm{ksec}$. A broadband $(0.3-200 \mathrm{keV})$ spectral analysis reveals that the source continuum spectrum is well-fitted by a blackbody $\left(k T_{\mathrm{BB}} \simeq\right.$ $0.56 \mathrm{keV}$ ), contributing $\sim 14 \%$ of the observed $0.1-200 \mathrm{keV}$ flux, plus a Comptonized component modeled by comptt (seed photon temperature of $\sim 1.1 \mathrm{keV}$, electron temperature $\sim 2.7 \mathrm{keV}$, and optical depth $\sim 11$ ), plus a hard power-law component (photon index $\sim 2.9$ ) contributing about $11 \%$ of the $0.1-200 \mathrm{keV}$ source flux. An emission line at $6.4-6.7 \mathrm{keV}$ is also significantly detected. This feature is quite broad and, in line with previous works (Di Salvo et al. 2005), we fitted it with a diskline model, yielding an inner disk radius of $\sim 8 R_{\mathrm{g}}\left(R_{\mathrm{g}}=G M / c^{2}=\right.$ $1.5 \mathrm{~km}$ for $M=1 M_{\odot}$ is the gravitational radius) and a relatively low inclination of about $30^{\circ}$. The $0.1-200 \mathrm{keV}$ source luminosity was $3.8 \times 10^{37} \mathrm{erg} \mathrm{s}^{-1}$ assuming a distance to the source of $7.4 \mathrm{kpc}$ (Haberl \& Titarchuk 1995).

The persistent emission spectrum of $4 \mathrm{U} 1705-44$ has been discussed by Barret \& Olive (2002) and recently by Di Salvo et al. (2005). Barret \& Olive (2002) observed 4U 1705-44 with the Rossi X-Ray Timing Explorer during a clear spectral state transition (soft-hard-soft) and interpreted the observed spectral evolution of the source within a scenario in which the accretion geometry is made of a truncated accretion disk of varying radius and an inner flow merging smoothly with the neutron star boundary layer (Barret et al. 2000; Done 2002). Barret \& Olive (2002) show that the truncation radius is not set by the instantaneous accretion rate, as observations with the same bolometric luminosity may have very different spectral and timing properties. A possible mechanism for the state transition is the evaporation of the disk due to the conduction of heat between the hot inner flow and the cold disk (Różańska \& Czerny 2000). The disk is a powerful source of cooling for the Comptonization; the closer the disk gets to the neutron star, the more effectively it cools the inner flow (Done 2002). In agreement with Barret \& Olive (2002), we interpret the soft (blackbody) component observed during the BeppoSAX observation as dominated by the disk emission

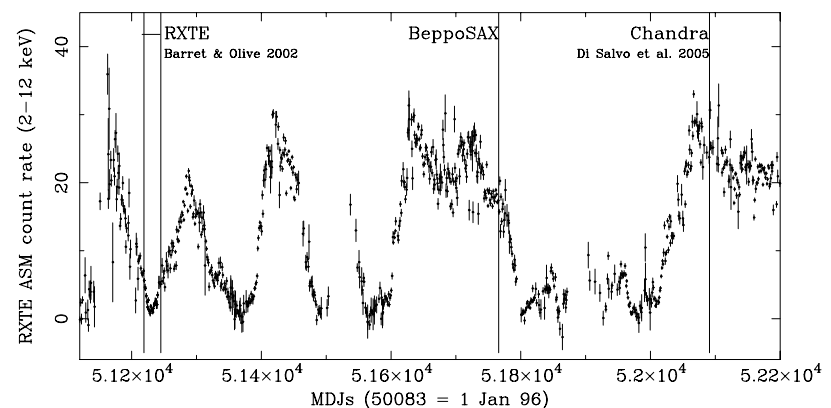

Fig. 3. Long-term RXTE/ASM 2-12 keV light curve (HEASERC public data base). The time of the BeppoSAX, RXTE, and Chandra observations are indicated with vertical lines.

at the highest luminosity (a contribution from the neutron star surface cannot be excluded). The fact that a simple blackbody provides a better fit to the soft component of the spectrum than the usual diskbb model may suggest that the disk differs from a standard thin disk because of the presence of a boundary layer. The Comptonized component probably originates from the (hotter) inner flow, since the seed photon and electron temperature of our best-fit model are higher than the temperature of the blackbody component detected in the spectrum. The high optical depth of the Comptonization component, $\tau \sim 11$, indicates that the source of the seed photons should lie inside the Comptonization region and not be directly visible as a component in the X-ray spectrum.

Di Salvo et al. (2005) observed 4U 1705-44 in the energy range $0.3-10 \mathrm{keV}$, with Chandra during a soft state and modeled the continuum of the energy spectrum with a soft Comptonization model, comptt (electron temperature $k T_{\mathrm{e}} \sim$ $2.3 \mathrm{keV}$ and optical depth $\tau \sim 18$ for a spherical geometry). The $(0.1-10 \mathrm{keV})$ luminosity was $3.3 \times 10^{37} \mathrm{erg} \mathrm{s}^{-1}$. Both the spectral parameters and the X-ray luminosity of the source during the Chandra observation are similar to the spectral parameters and luminosity during our BeppoSAX observation, suggesting that the source in both cases was in a soft spectral state. In particular, we probably observed the source in a soft/high spectral state in the lower banana state of the atoll-track. In the RXTEASM energy range, this observation was at the beginning of a transition between high rate and low rates and at a flux level intermediate between the ones reported by Di Salvo et al. (2005) and Barret \& Olive (2002) (Fig. 3).

Three discrete features (residuals from the continuum model fits) at energies below $3 \mathrm{keV}$ were fitted and identified in the Chandra X-ray spectrum of $4 \mathrm{U} 1705-44$. The iron $K \alpha$ line at $6.5 \mathrm{keV}$ was found to be intrinsically broad $(F W H M \sim 1.2 \mathrm{keV})$ and compatible with reflection from a cold accretion disk with $R_{\text {in }} \sim 15 \mathrm{~km}$ or with a Compton broadening in the external parts of a $\sim 2 \mathrm{keV}$ corona. A broad and strong emission line is also necessary to fit the BeppoSAX spectrum. In agreement with Di Salvo et al. (2005), we fit this feature with a diskline model, finding best-fit values of the spectral parameters that agree well with the Chandra results. In particular we find the line centroid energy at $6.7_{-0.5}^{+0.2} \mathrm{keV}$, compatible, within the uncertainty of the BeppoSAX spectral fitting, with the line energy of $6.4 \mathrm{keV}$ found from the Chandra observation. The main difference between our results and the Chandra results is in the best-fit value of the disk inclination, which is higher for Chandra $\left(59_{-4}^{+25} \mathrm{deg}\right)$ than for BeppoSAX. Indeed, if we fix the line centroid energy to $6.4 \mathrm{keV}$, the best-fit value from the Chandra observation, we find an inclination angle of the disk between $20^{\circ}$ and $50^{\circ}$, barely compatible with the Chandra result. 
In this Letter, we report, for the first time, the presence of a hard X-ray (power-law shaped) component in the soft state of $4 \mathrm{U} 1705-44$, with a photon index of $\sim 2.9$ contributing $\sim 11 \%$ of the observed source flux. Hard-energy tails, steep power-laws with photon indexes $\sim 2-3$ and without evidence of a high energy cutoff are observed in the spectra of accreting black hole candidates (BHCs) during the intermediate state (IS) or very high state (VHS) ${ }^{1}$. This component is variable and usually contributes a few per cent of the total flux in the IS state, while in the VHS (or SPL) state it contributes up to $40-90 \%$ of the total flux (Remillard \& McClintock 2006). On the other hand, recent broad band studies have shown that the spectra of most Zsources display a variable hard X-ray component (e.g. Di Salvo et al. 2000, 2001, 2002; Paizis et al. 2006). In some cases, it has been shown that the presence of this hard component is correlated with the source spectral state. Specifically, the power-law component is the strongest at the lowest inferred mass accretion rates. For the highest mass accretion rates of Z-sources (for instance in the flaring branch of the CD) and in the high state, HS, of BHCs, the intensity of the hard X-ray tail is often below detectability.

This suggests a similarity between the spectrum of the atoll source 4U 1705-44 during this soft banana state and the spectra of BHCs and Z-sources during some spectral states, such as IS/VHS and horizontal and perhaps normal branch, respectively. Indeed, other LMXBs of the atoll class have shown the presence of a similar hard X-ray component (see e.g. Paizis et al. 2006 for the bright atoll source GX 13+1; Fiocchi et al. 2006 for $4 \mathrm{U}$ 1636-53; Tarana et al. 2007 for $4 \mathrm{U}$ 1820-30). We conclude that these hard X-ray components are becoming more and more ubiquitous among X-ray binaries, indicating that similar emission mechanisms and geometry characterizes all these systems. As proposed for Z-sources, the hard tails in the soft state of $4 \mathrm{U} 1705-44$ can be produced either in a hybrid thermal/non thermal model (Poutanen \& Coppi 1998) or in a bulk motion of matter close to the neutron star (e.g. Titarchuk \& Zannias 1998). Alternative mechanisms that have been proposed is Comptonization of seed photons by relativistic electrons in a jet (e.g Di Salvo et al. 2000) and synchrotron emission from a relativistic jet escaping the system (Markoff et al. 2001).
Acknowledgements. This work was partially supported by the German Space Agency (DLR) under contracts nos. 50 OG 9601 and 50 OG 0501, by the Consiglio Nazionale delle Ricerche and by the Ministero della Istruzione, della Universitá e della Ricerca (MIUR).

\section{References}

Arnaud, K. A. 1996, Astronomical Data Analysis Software and Systems V, ed. G. H. Jacoby \& J. Barnes, ASP Conf. Ser., 101, 17

Barret, D., \& Vedrenne, G 1994, ApJS, 92, 505

Barret, D., \& Olive, J. F. 2002, ApJ, 576, 391

Barret, D., Olive, J. F., Boirin, L. et al. 2000, ApJ, 533, 329

Boella, G., Butler, R. C, Perola, G. C., et al. 1997a, A\&AS, 122, 299

Boella, G., Chiappetti, L., Conti, G., et al. 1997b, A\&AS, 122, 327

Di Salvo, T., \& Stella, L. 2002, Proc. of the XXIIth Rencontres de Moriond, ed. A. Goldwurm, D. N. Neumann \& J. Tran Thanh Van, [arXiv: astro-ph/0207219]

Di Salvo, T., Stella, L., Robba, N. R. et al. 2000, ApJ, 544, 119

Di Salvo, T., Robba, N. R., Iaria, R. et al. 2001, ApJ, 544, 49

Di Salvo, T., Iaria, R., Méndez, M., et al. 2005, ApJ, 623, L121

Done, C. 2002, Philos. Trans. R. Soc. London A., 360 (1798), 1967

Fabian, A. C., Rees, M. J., Stella, L., et al. 1989, MNRAS, 238, 729

Fiocchi, M., Bazzano, A., \& Ubertini, P. 2006, ApJ, 651, 416

Fiocchi, M., Bazzano, A., \& Ubertini, P. 2007, ApJ, 657, 448

Fiore, F., Guainazzi, M., \& Grandi, P. 1999, Cookbook for BeppoSAX NFI Spectral Analysis

Frontera, F., Costa E., Dal Fiume D., et al. 1997, A\&AS, 122, 357

Gierlínski, M., \& Done, C. 2002, MNRAS, 331, L47

Haberl, F., \& Titarchuk, L. 1995, A\&A, 299, 414

Hasinger, G., \& van der Klis, M. 1989, A\&A, 225, 79

Langmeier, A., Sztajno, M., Hasinger, G. et al. 1987, ApJ, 323, 288

Manzo, G., Giarrusso, S., Santangelo, A., et al. 1997, A\&AS, 122, 341

Markoff, S., Falcke, H., \& Fender, R. 2001, A\&A, 372, L25

Muno, M. P., Remillard, R., \& Chakrabarti, D. 2002, ApJ, 568, L35

Parmar, A. N., Martin, D. D.E, Bavdaz, M., et al. 1997, A\&AS, 122, 309

Paizis, A., Farinelli, R., Titarchuk, L. et al. 2006, A\&A, 459, 187

Piraino, S., Santangelo, A., \& Kaaret, P. 2000, A\&A, 360, L35

Piraino, S., et al. 2007, in preparation

Poutanen, J., \& Coppi, P. S. 1998, Phys. Scr., T77, 57

Remillard R. A., \& McClintock, J. E. 2006, ARA\&A, 44, 49

Różańska, A., \& Czerny, B. 2000, MNRAS, 316, 473

Sztajno, M.,Langmeier, A., Frank, J. et al. 1985, IAU Circ, 4111

Tanaka, Y., \& Lewin W. H. G. 1995, X-ray Binaries (Cambridge University Press), 252

Tarana, A., Bazzano, A., Ubertini, P. et al. 2007, ApJ, 654, 494

Titarchuk, L. 1994, ApJ, 434, 570

Titarchuk, L., \& Zannias, T. 1998, ApJ, 493, 863

van der Klis, M. 2006, Compact Stellar X-Ray Sources, ed. W. H.G Lewin \& van der Klis, 39

1 Note that Remillard \& McClintock (2006) call the VHS state the steep power law (SPL) state. 